\title{
ANALYSIS OF ULTRASHORT LASER PULSE IRRADIATION WITH 2D THIN METAL FILMS USING THE FUZZY LATTICE BOLTZMANN METHOD
}

\author{
Alicja Piasecka-Belkhayat, Anna Korczak \\ Silesian University of Technology, Institute of Computational Mechanics and Engineering, Gliwice, Poland \\ e-mail: alicja.piasecka-belkhayat@polsl.pl; anna.korczak@polsl.pl
}

This paper presents the numerical modelling of heat transfer in two-dimensional metal films. The mathematical model of the problem analyzed consists on fuzzy coupled lattice Boltzmann equations for electrons and phonons supplemented by adequate boundary-initial conditions. In this model, the standard two-dimensional 9-speed lattice (D2Q9) is used. The main concept behind this work was to use the fuzzy lattice Boltzmann method (FLBM) to analyze the thermal process proceeding in a thin metal film. The application of $\alpha$-cuts allows one to simplify mathematical operations in the fuzzy numbers set. Additionally, the trapezoidal approximation of fuzzy relaxation times and boundary conditions is considered. In the final part of the paper, the results of numerical computations are shown.

Keywords: heat transfer, Boltzmann transport equation, BTE, fuzzy arithmetic

\section{Nomenclature}

e $\quad-$ energy density $\left[\mathrm{J} / \mathrm{m}^{3}\right]$

$G \quad-$ electron-phonon coupling factor (characterizes energy exchange between carriers) $\left[\mathrm{W} /\left(\mathrm{m}^{3} \mathrm{~K}\right)\right]$

$I_{0} \quad-$ peak power intensity of laser pulse $\left[\mathrm{W} / \mathrm{m}^{2}\right]$

$k_{b} \quad-$ Boltzmann constant $[\mathrm{J} / \mathrm{K}]$

$n_{e} \quad-$ electron density $\left[1 / \mathrm{m}^{3}\right]$

$r \quad-$ radius of laser beam

$Q \quad-$ energy source related to unit volume $\left[\mathrm{W} / \mathrm{m}^{3}\right]$

$Q^{\prime} \quad-\quad$ power density deposited by external source function associated with laser irradiation $\left[\mathrm{W} / \mathrm{m}^{3}\right]$

$t, \Delta t \quad-$ time and time increment $[\mathrm{ps}]$

$T \quad-$ temperature $[\mathrm{K}]$

$\mathbf{v} \quad-$ frequency-dependent propagation speed $[\mathrm{m} / \mathrm{s}]$

$X, Y \quad-$ spacial coordinate corresponding to $x$ and $y$ axis $[\mathrm{nm}]$

$\Delta x, \Delta y-$ spacial increment corresponding to $x$ and $y$ axis [nm]

$\beta-$ laser pulse parameter $[1 / \mathrm{s}]$

$\delta \quad-$ absorption coefficient $[1 / \mathrm{nm}]$

$\begin{array}{lll}\varepsilon_{F} & - & \text { Fermi energy }[\mathrm{J}]\end{array}$

$\eta \quad-$ number density of oscillators $\left[1 / \mathrm{m}^{3}\right]$

$\theta_{D} \quad-$ Debye temperature $[\mathrm{K}]$

$\tau_{r} \quad-$ relaxation time $[\mathrm{ps}]$

Subscripts: $e-$ electron, $p h-$ phonon; superscript 0 - equilibrium; and $\sim$ - fuzzy 


\section{Introduction}

A very important problem is the correct estimation of heat transfer processes occurring in thin metal films subjected to an ultrafast laser pulse in microtechnical applications. Extreme temperature gradients, very short duration of the laser irradiation process and small dimensions of the domain considered make it impossible to use the macroscopic heat conduction equation based on Fourier's law (Bejan and Kraus, 2003). It is possible to introduce a relaxation time, i.e. delay time (time interval) between the heat flux and temperature gradient, and obtain a generalization of the Fourier law called the Cattaneo-Vernotte equation (Cattaneo, 1958). Another possibility is the dual phase lag equation (DPLE) containing two delay times: relaxation time of the heat flux vector and relaxation time of the temperature gradient. This equation contains a second order time derivative and a higher order mixed derivative in time and space (Majchrzak and Mochnacki, 2009, 2014; Majchrzak and Kałuża, 2015; Mochnacki and Paruch, 2013; Zhang, 2007; Chen et al., 2004). The two-temperature model (hyperbolic or parabolic) consisting of equations describing temporal and spatial evolution of lattice and electrons temperatures together with lattice and electron heat fluxes can also be used to solve this type of heat transfer problems (Ho et al., 2003; McDonough et al., 2006; Majchrzak and Dziatkiewicz, 2015; Tzou, 1997).

In the paper, the fuzzy lattice Boltzmann method (Piasecka-Belkhayat and Korczak, 2014a,b) is used to analyze thermal processes occurring in thin metal films subjected to an ultrafast laser pulse. In the engineering practice, thermal processes are generally analyzed with the assumption that the equations describing the analyzed process and material parameters are deterministic. The solution of the classical lattice Boltzmann method (LBM) does not give an exact image of natural phenomena because some of material parameters are determined experimentally. It seems natural to consider fuzzy material parameters occurring in the mathematical description. The application of the FLBM allows one to include in the mathematical model "uncertainty" associated with material parameters. Unlike exact sets, where the membership function can take only two values: 0 or 1 , in the fuzzy set theory a partial membership to the fuzzy set is considered. In the paper, fuzzy values of relaxation times and boundary conditions are taken into account. The closed intervals called $\alpha$-cuts and an algebraic extension of the classical interval arithmetic called the directed interval arithmetic were used to solve the problem considered (Piasecka-Belkhayat and Korczak, 2016; Mochnacki and Piasecka-Belkhayat, 2013). The use of directed interval arithmetic allows one to obtain narrower temperature intervals than in the case of the classical interval arithmetic, while the $\alpha$-cuts make it possible to perform mathematical operations in a set of "normal" intervals, which is much simpler. Trapezoidal fuzzy numbers along with triangular numbers are the most commonly used fuzzy numbers. It is possible to use a non-linear membership function (for example Gaussian membership function) but this does not have a significant impact on the obtained solution.

During laser-metal interaction, the energy transfer process of excited electrons to phonons via coupling between both energy carriers can be observed. The Boltzmann transport equations transformed into a form using carrier energy densities for the coupled model can be written in the following form

$$
\frac{\partial e_{e}}{\partial t}+\mathbf{v}_{e} \cdot \nabla e_{e}=-\frac{e_{e}-e_{e}^{0}}{\tau_{r e}}+Q_{e} \quad \frac{\partial e_{p h}}{\partial t}+\mathbf{v}_{p h} \cdot \nabla e_{p h}=-\frac{e_{p h}-e_{p h}^{0}}{\tau_{r p h}}+Q_{p h}
$$

where $e$ means electrons and $p h$ means phonons.

The above energy equations must be supplemented by appropriate boundary and initial conditions.

The electron and phonon energy densities depending on the lattice temperatures are calculated using the formulas (Mochnacki and Paruch, 2013) 


$$
e_{e}\left(T_{e}\right)=\left(n_{e} \frac{\pi^{2}}{2} \frac{k_{b}^{2}}{\varepsilon_{F}}\right) T_{e}^{2} \quad e_{p h}\left(T_{p h}\right)=\left(\frac{9 \eta_{p h} k_{b}}{\theta_{D}^{3}} \int_{0}^{\theta_{D} / T_{p h}} \frac{z^{3}}{\exp (z)-1} d z\right) T_{p h}^{4}
$$

while the electron and phonons source functions defined per unit volume are expressed by the following equations (Ghai et al., 2005; Venkatakrishnan et al., 2002; Lee et al., 2011)

$$
Q_{e}=Q^{\prime}-G\left(T_{e}-T_{p h}\right) \quad Q_{p h}=G\left(T_{e}-T_{p h}\right)
$$

\section{The fuzzy Boltzmann transport equation}

Assuming fuzzy values of parameters occurring in equations (1.1), a fuzzy version of the Boltzmann transport equations is obtained (Ghai et al., 2005)

$$
\begin{aligned}
& \frac{\partial \widetilde{e}_{e}(t, x, y)}{\partial t}+\mathbf{v}_{e} \cdot \nabla \widetilde{e}_{e}(t, x, y)=-\frac{\widetilde{e}_{e}(t, x, y)-\widetilde{e}_{e}^{0}(t, x, y)}{\widetilde{\tau}_{r e}}+\widetilde{Q}_{e}(t, x, y) \\
& \frac{\partial \widetilde{e}_{p h}(t, x, y)}{\partial t}+\mathbf{v}_{p h} \cdot \nabla \widetilde{e}_{p h}(t, x, y)=-\frac{\widetilde{e}_{p h}(t, x, y)-\widetilde{e}_{p h}^{0}(t, x, y)}{\widetilde{\tau}_{r p h}}+\widetilde{Q}_{p h}(t, x, y)
\end{aligned}
$$

The fuzzy values of the electron and phonon temperatures for their equivalent energy densities are given by the formulas

$$
\widetilde{T}_{e}=\sqrt{\widetilde{e}_{e}\left(\widetilde{T}_{e}\right) /\left(n_{e} \frac{\pi^{2}}{2} \frac{k_{b}^{2}}{\varepsilon_{F}}\right)} \quad \widetilde{T}_{p h}=\sqrt[4]{\widetilde{e}\left(\widetilde{T}_{p h}\right) \theta_{D}^{3} /\left(9 \eta k_{b} \int_{0}^{\theta_{D} / \widetilde{T}_{p h}^{f-1}} \frac{z^{3}}{\exp (z)-1} d z\right)}
$$

whereas the fuzzy electron and phonon energy sources are calculated from the following formulas (Ghai et al., 2005)

$$
\begin{aligned}
& \widetilde{Q}_{e}(t, x, y)=Q^{\prime}(t, x, y)-G\left[\widetilde{T}_{e}(t, x, y)-\widetilde{T}_{p h}(t, x, y)\right] \\
& \widetilde{Q}_{p h}(t, x, y)=G\left[\widetilde{T}_{e}(t, x, y)-\widetilde{T}_{p h}(t, x, y)\right]
\end{aligned}
$$

The source component $Q^{\prime}(t, x, y)$ appearing in the electron energy equation determining temporary changes of the laser output pulse can be approximated using the exponential function defined as follows (Chen et al., 2006)

$$
Q^{\prime}(t, x, y)=I_{0} \delta \exp (-\delta y-\beta t) \exp \left(-\frac{2 x^{2}}{r^{2}}\right)
$$

In the present paper, the D2Q9 (two-dimensional nine-velocity lattice type) has been employed. For this lattice model, the discrete velocities for electrons and phonons (Fig. 1) can be expressed as

$$
\mathbf{v}_{d}= \begin{cases}{[0,0]} & \text { for } \quad d=0 \\ {\left[\cos \frac{(d-1) \pi}{2}, \sin \frac{(d-1) \pi}{2}\right]} & \text { for } \quad d=1, \ldots, 4 \\ \sqrt{2}\left[\cos \left(\frac{(d-5) \pi}{2}+\frac{\pi}{4}\right), \sin \left(\frac{(d-5) \pi}{2}+\frac{\pi}{4}\right)\right] c & \text { for } \quad d=5, \ldots, 8\end{cases}
$$

where $c=\Delta x / \Delta t=\Delta y / \Delta t$ is the lattice speed uniform in both directions, the time step $\Delta t=t^{f+1}-t^{f}$ is recommended to be shorter than the carriers relaxation time (Escobar et al., 2006).

The main steps of numerical analysis summarizing the presented method are shown in the flow chart below (Fig. 2). 


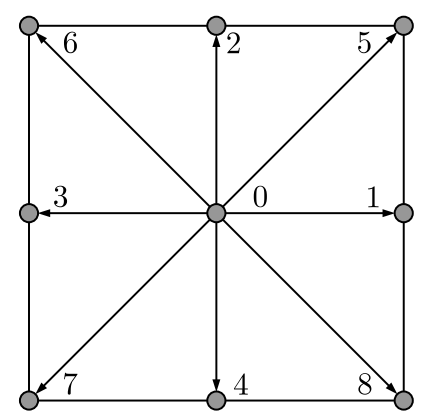

Fig. 1. Directions of carriers velocities in the two-dimensional 9-speed (D2Q9) lattice Boltzmann model

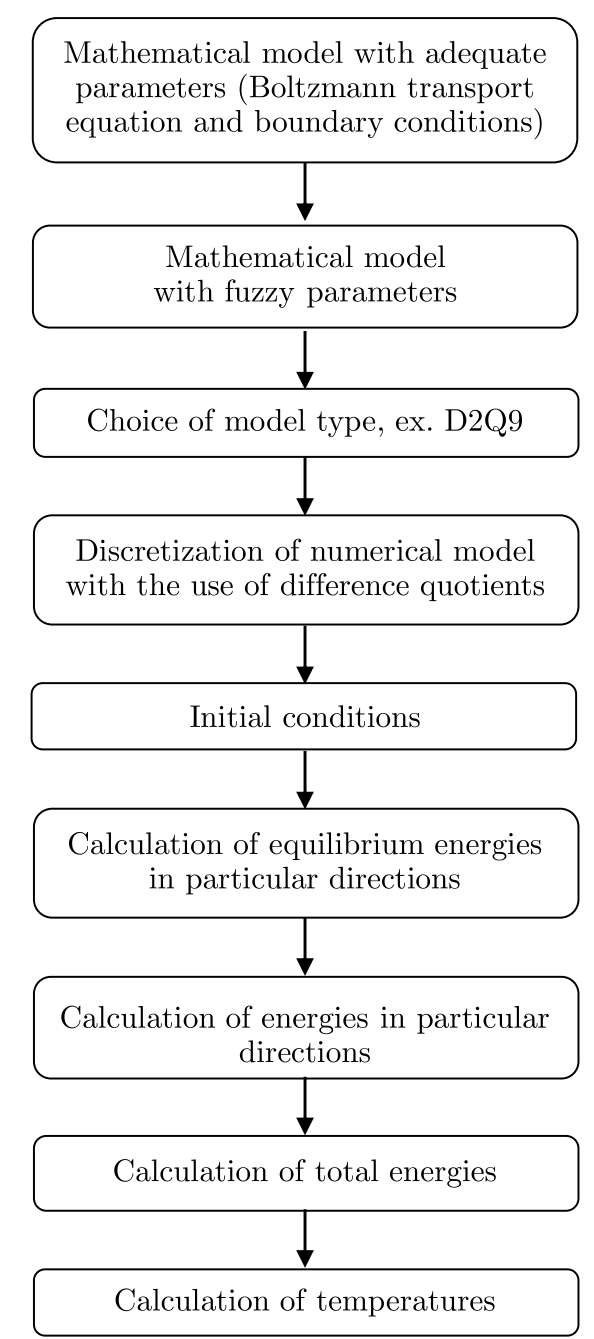

Fig. 2. Flow chart of the main steps in the algorithm

\section{Results of computations}

As a numerical example, the heat transport in a gold thin film of dimensions $1000 \mathrm{~nm} \times 200 \mathrm{~nm}$ has been analysed. The following input data have been introduced: $\widetilde{q}_{b 1}=\widetilde{q}_{b 2}=\widetilde{q}_{b 3}=\widetilde{0} \mathrm{~W} / \mathrm{m}^{2}$, fuzzy trapezoidal boundary temperature $\widetilde{T}_{b 4}=(285,292.5,307.5,315) \mathrm{K}, T_{0}=300 \mathrm{~K}, \Delta t=$ $0.01 \mathrm{ps}$, fuzzy trapezoidal relaxation times for phonons $\widetilde{\tau}_{p h}=\left(\tau_{p h}-0.05 \tau_{p h}, \tau_{p h}-0.025 \tau_{p h}, \tau_{p h}+\right.$ $\left.0.025 \tau_{p h}, \tau_{p h}+0.05 \tau_{p h}\right)$ and electrons $\widetilde{\tau}_{e}=\left(\tau_{e}-0.05 \tau_{e}, \tau_{e}-0.025 \tau_{e}, \tau_{e}+0.025 \tau_{e}, \tau_{e}+0.05 \tau_{e}\right)$, $r=160 \mathrm{~nm}$, the other material and laser properties are defined in Table 1 . In the paper, the fuzzy heat fluxes are calculated numerically with the use of the rules of fuzzy analysis. 
Table 1. Material and laser properties

\begin{tabular}{|c|c|c|c|c|c|c|c|c|}
\hline & $\begin{array}{c}\tau_{p h} \\
{[\mathrm{ps}]}\end{array}$ & $\begin{array}{c}\tau_{e} \\
{[\mathrm{ps}]}\end{array}$ & $\begin{array}{c}\theta_{D} \\
{[\mathrm{~K}]}\end{array}$ & $\begin{array}{c}n_{e}\left(\cdot 10^{28}\right) \\
{\left[1 / \mathrm{m}^{3}\right]}\end{array}$ & $\begin{array}{c}\varepsilon_{F} \\
{[\mathrm{eV}]}\end{array}$ & $\begin{array}{c}I_{0}\left(\cdot 10^{13}\right) \\
{\left[\mathrm{W} / \mathrm{m}^{2}\right]}\end{array}$ & $\begin{array}{c}\beta\left(\cdot 10^{13}\right) \\
{[1 / \mathrm{s}]}\end{array}$ & $\begin{array}{c}\delta\left(\cdot 10^{13}\right) \\
{[1 / \mathrm{m}]}\end{array}$ \\
\hline \hline $\mathrm{Au}$ & 0.8 & 0.04 & 170 & 5.9 & 5.53 & 2 & 0.5 & 7.55 \\
\hline
\end{tabular}

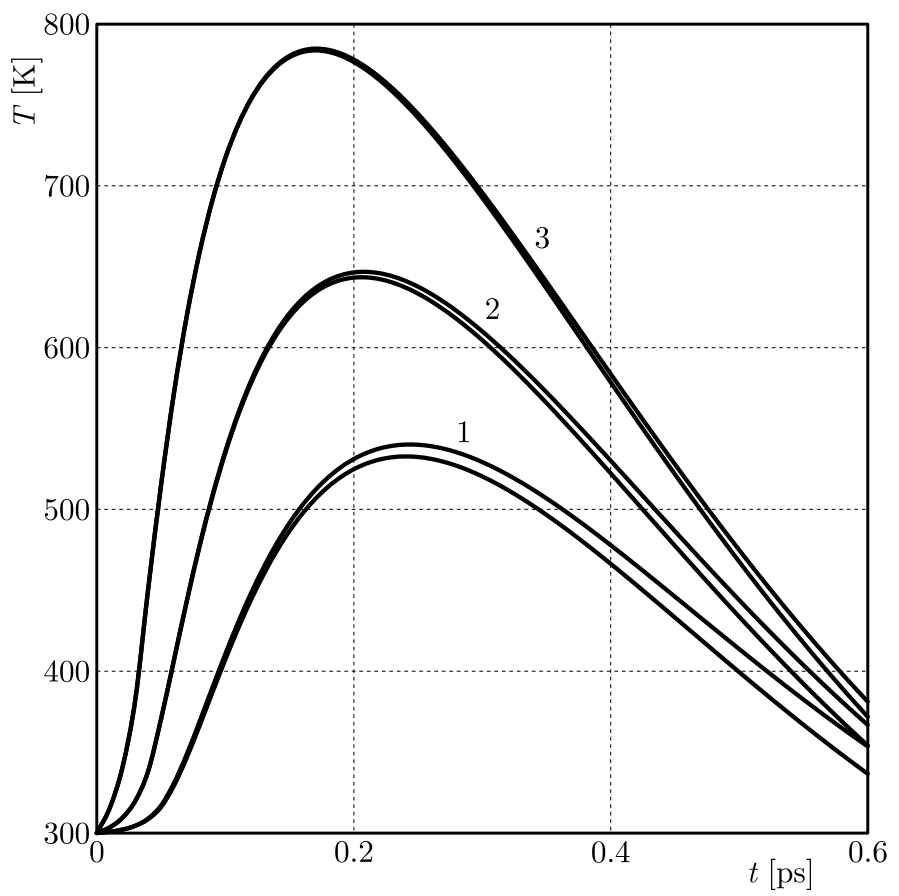

Fig. 3. Fuzzy electrons heating curves for chosen nodes for $\alpha=0.5$

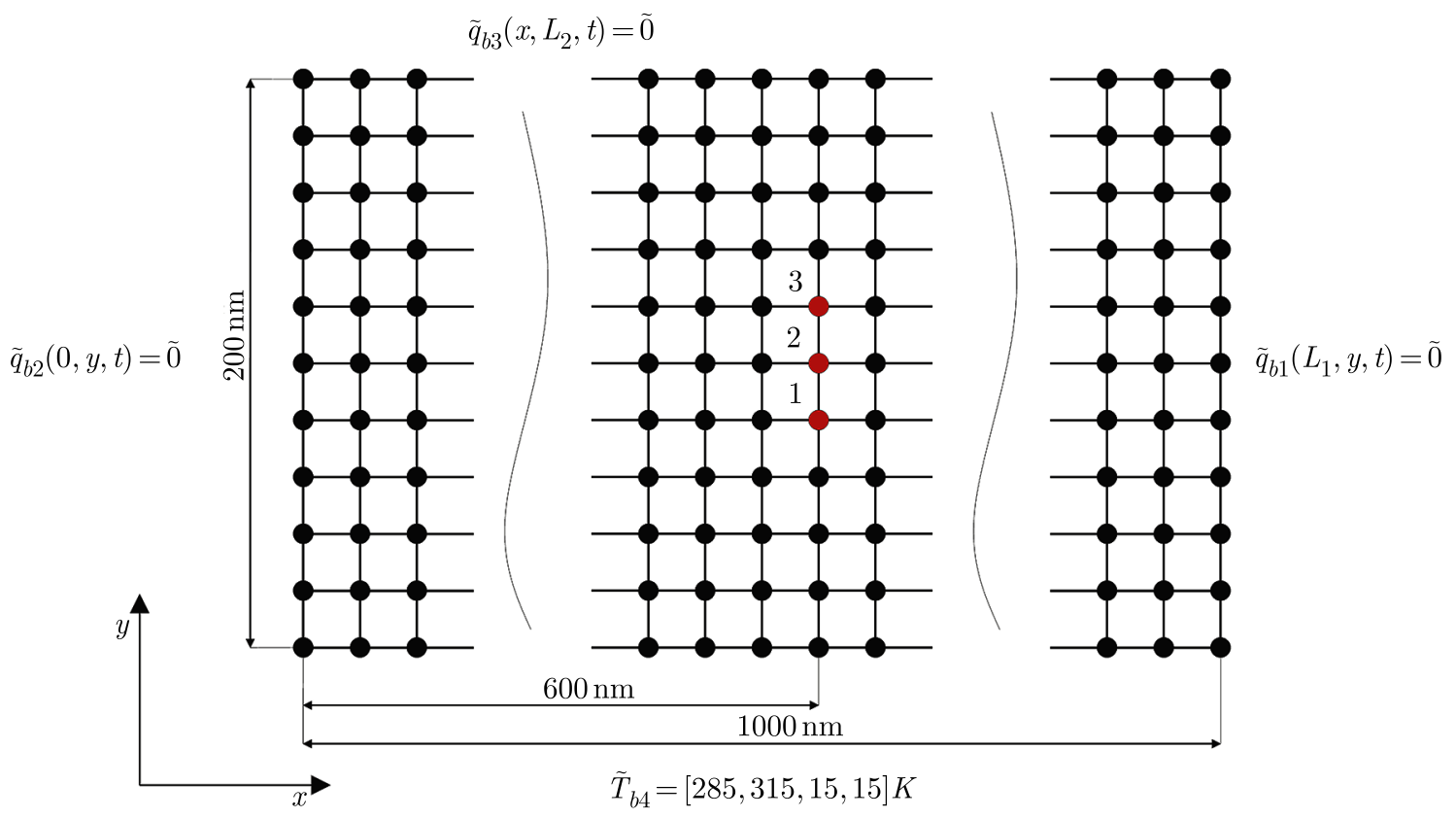

Fig. 4. Lattice with marked analysed nodes 
Figure 3 illustrates the fuzzy electrons heating curves obtained for $\alpha=0.5$ in the nodes: $(100,80)-1,(100,100)-2$ and $(100,120)-3$ (see Fig. 4$)$. For each node we have two curves, they are graphs of the beginnings and ends of $\alpha$-cuts of temperatures, which are intervals.

In the second example, the temperature distribution for nodes with the $O X$ coordinate equal to $600 \mathrm{~nm}$ was analyzed (Fig. 6). Results after $0.5 \mathrm{ps}, 0.7 \mathrm{ps}$ and $0.9 \mathrm{ps}$ also for $\alpha=0$ and $\alpha=0.5$ are shown in Fig. 5. What can be seen, for bigger alpha we have narrower temperature intervals.
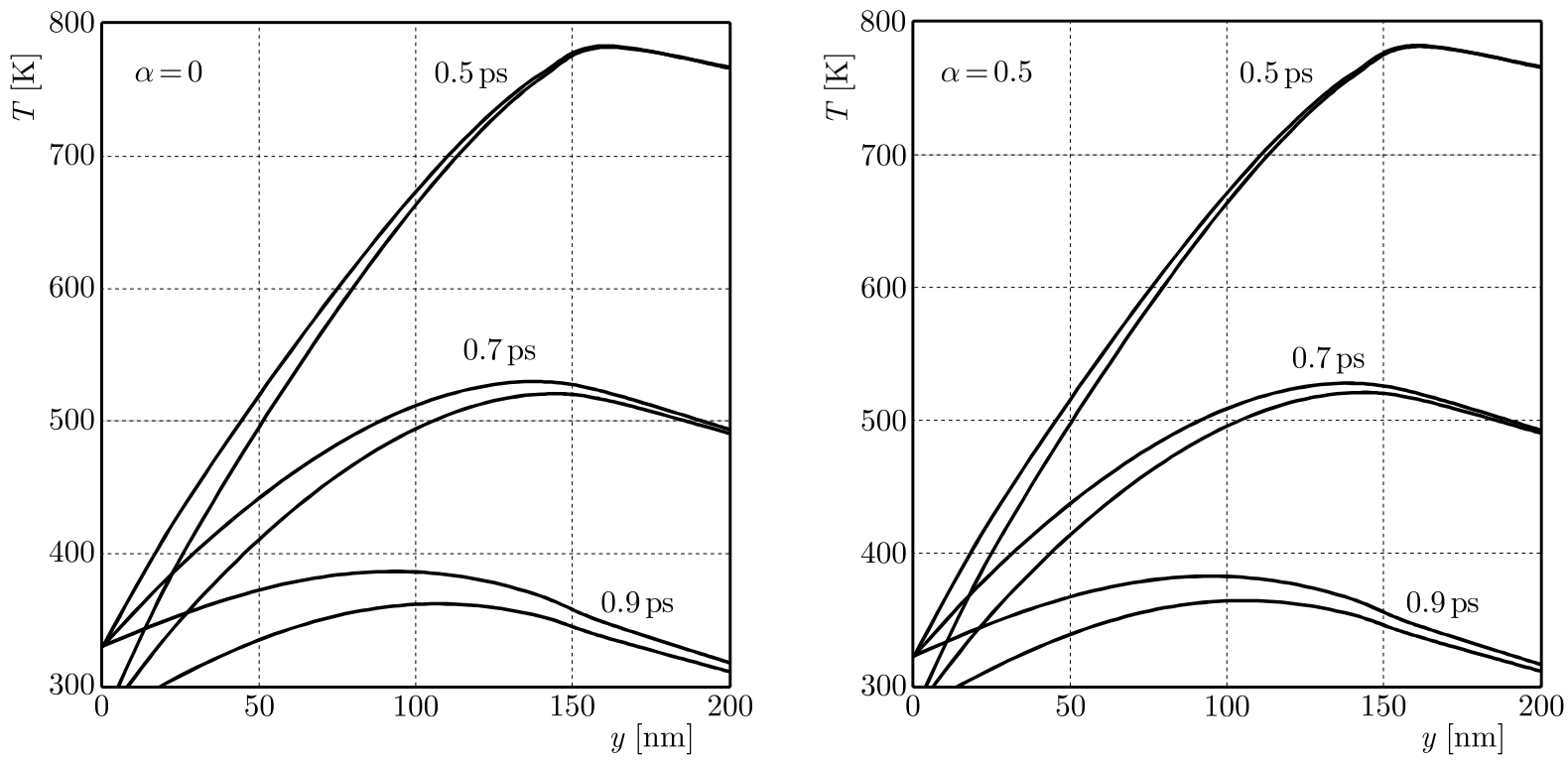

Fig. 5. Fuzzy electron temperature distribution for $\alpha=0$ and $\alpha=0.5$

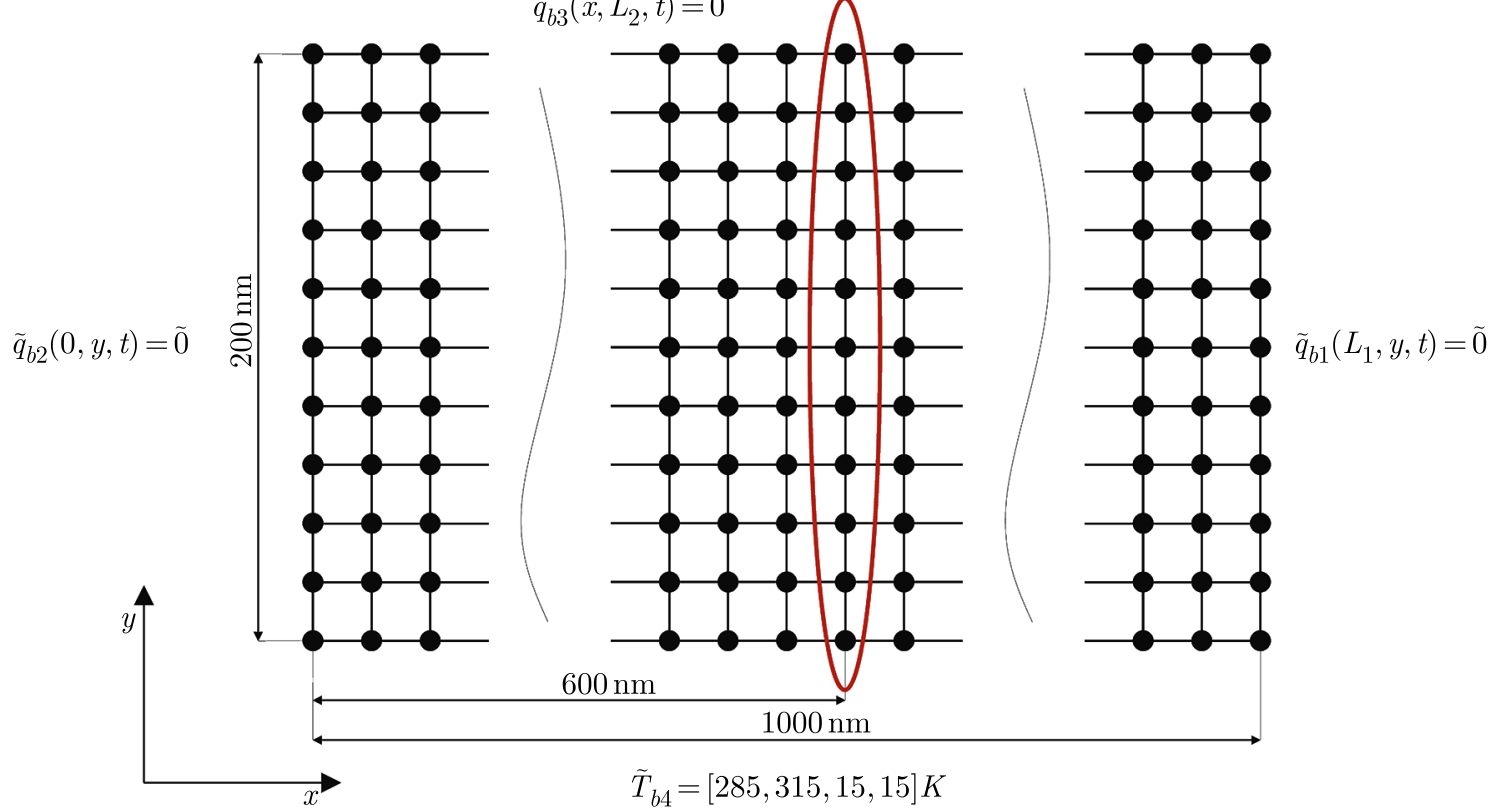

Fig. 6. Lattice with marked analysed nodes 
The third example (Fig. 7) illustrates the result of calculations obtained at one node $(500,60) \mathrm{nm}$ after $0.9 \mathrm{ps}$, which is a trapezoidal fuzzy number with marked particular $\alpha$-cuts.

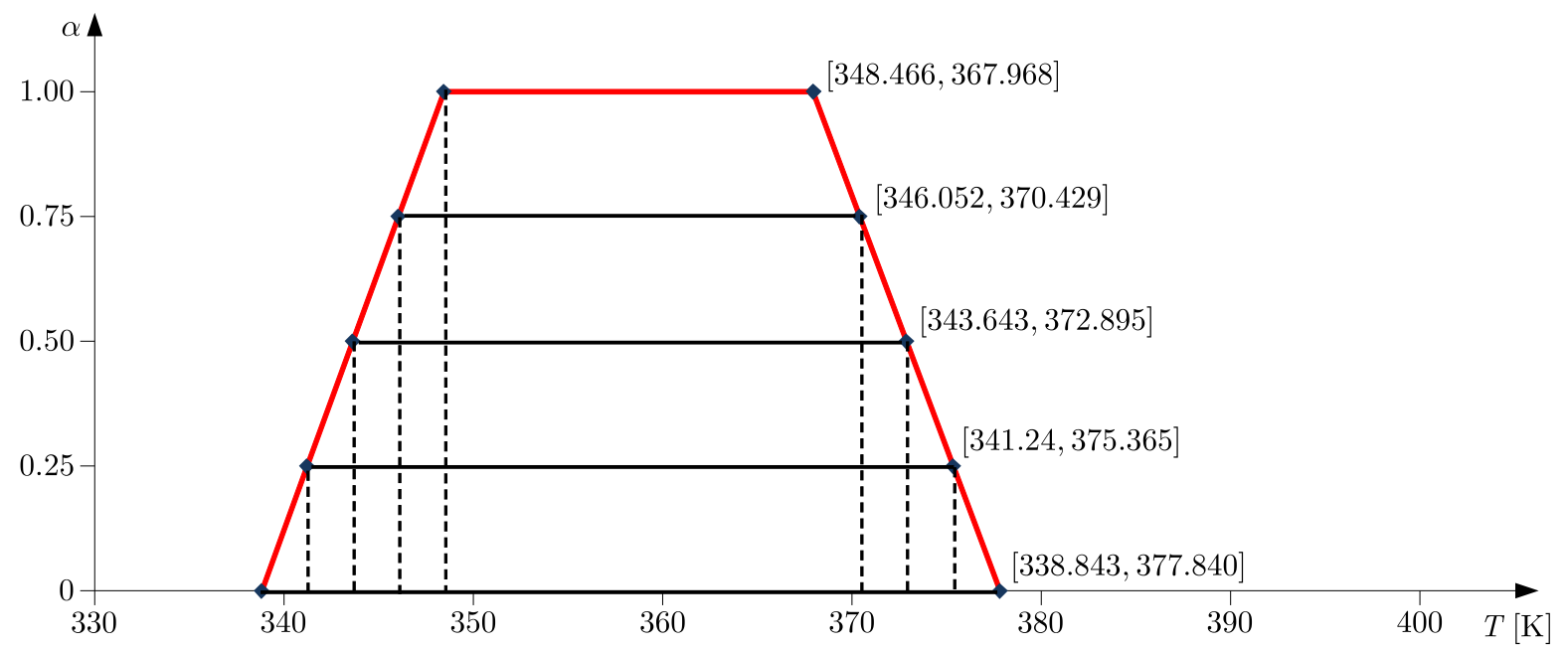

Fig. 7. Trapezoidal fuzzy number - temperature at the chosen node $(500,60) \mathrm{nm}$ after $0.9 \mathrm{ps}$

\section{Conclusions}

In this study, the numerical modelling of heat transfer in two-dimensional metal films has been considered. The main idea of this article was to include in the mathematical model fuzzy values of some parameters, for example relaxation times and boundary conditions.

The fuzzy lattice Boltzmann method has been applied to solve the problem discussed defined in this way. It should be also pointed out that the $\alpha$-cuts have been used in order to avoid complicated calculations in the set of fuzzy numbers. The application of $\alpha$-cuts allows one to consider fuzzy numbers as interval numbers and use simpler interval arithmetic. The trapezoidal approximation of fuzzy relaxation times and boundary conditions has been considered. As shown in Fig. 6 , the bigger values of the parameter $\alpha$ cause the narrower temperature interval, whereas for $\alpha$ equal to 1 the width of the temperature interval is equal to 0 .

The presented fuzzy LBM allows one to find the numerical solution in the fuzzy form, which is particularly important in the case of experimentally estimated parameters, e.g. the relaxation time. It is worth to emphasize that the classical error analysis is not used in numerical methods based on the interval or fuzzy arithmetic. However, a very effective tool to control the width of intervals obtained as a result of calculations is the sensitivity analysis (Piasecka-Belkhayat and Korczak, 2017). Another important aspect is that the results obtained with the use of the classical lattice Boltzmann method for exact values should be included in the middle of intervals obtained with the use of fuzzy LBM (Piasecka-Belkhayat and Korczak, 2014).

\section{A. Appendix - fuzzy arithmetic}

In this paper, all calculations have been performed using the fuzzy set theory. This kind of arithmetic is not so common in mathematical modelling, especially in solving heat transfer problems, and that is the reason why some of the rules and definitions of the fuzzy arithmetic ground must be explained (Hanss, 2005). 
At the beginning, it is necessary to introduce the definition of a fuzzy set. Let the fuzzy set $\widetilde{C}$ be a nonempty subset of the universal set $\mathbb{X}(\widetilde{C} \subseteq \mathbb{X})$, which can be defined by a set of pairs consisting of elements $x \in \mathbb{X}$ and a characteristic function $\mu_{\widetilde{C}}(x)$ called a membership function of the form

$$
\widetilde{C}=\left\{\left(x, \mu_{\widetilde{C}}(x)\right) ; x \in \mathbb{X}\right\}
$$

where the membership function $\mu_{\widetilde{C}}(x)$ can be formally expressed as

$$
\mu_{\widetilde{C}}(x): \quad \mathbb{X} \rightarrow[0,1]
$$

In the theory of fuzzy sets, each element from the set is mapped to the interval $[0,1]$ by a membership function. The interval $[0,1]$ means real numbers between 0 and 1 (including 0,1 ). Consequently, a fuzzy set is a "vague boundary set" compared with a crisp set.

For every $x \in \mathbb{X}$, three types of membership to the fuzzy set $\widetilde{C}$ can be defined (see Table 2 ).

Table 2. Membership to the fuzzy set

\begin{tabular}{|c|c|}
\hline$\mu_{\widetilde{C}}(x)$ & Remark \\
\hline \hline 1 & full membership to the fuzzy set, $x \in \widetilde{C}$ \\
\hline 0 & lack of membership to the fuzzy set, $x \notin \widetilde{C}$ \\
\hline$(0,1)$ & partial membership to the fuzzy set \\
\hline
\end{tabular}

Among the infinite quantity of possible fuzzy sets that can be treated as fuzzy numbers, some types of membership functions are of particular importance. In general, arithmetic operations on fuzzy numbers are very complicated. One of the ways to avoid difficult arithmetic operations performed on fuzzy numbers is to apply $\alpha$-cuts. The concept of $\alpha$-cuts is one of the most important concepts of fuzzy sets. The $\alpha$-cut of a set $\widetilde{C}$ in a universal set can be defined as a crisp set composed of members whose membership is not less than $\alpha$ for every $\alpha \in[0,1]$ (Otto et al., 1993)

$$
\widetilde{C}_{\alpha}=\left\{x \in \mathbb{X}: \quad \mu_{\widetilde{C}}(x) \geqslant \alpha\right\}
$$

It should be pointed out that the value $\alpha$ is arbitrary, and the characteristic function of the $\alpha$-cut set can be defined as follows

$$
\chi_{\widetilde{C}_{\alpha}}= \begin{cases}1 & \text { for } \quad \mu_{\widetilde{C}}(x) \geqslant \alpha \\ 0 & \text { for } \quad \mu_{\widetilde{C}}(x)<\alpha\end{cases}
$$

It is worth to emphasise that every fuzzy set $\widetilde{C}$ is exactly a sum of all its $\alpha$-cuts

$$
\widetilde{C}=\sum_{\alpha \in[0,1]} \alpha \widetilde{C}_{\alpha}
$$

where $\alpha \widetilde{C}_{\alpha}$ means a fuzzy set with the following membership function

$$
\mu_{\alpha \widetilde{C}_{\alpha}}=\left\{\begin{array}{lll}
\alpha & \text { for } & x \in \widetilde{C}_{\alpha} \\
0 & \text { for } & x \notin \widetilde{C}_{\alpha}
\end{array}\right.
$$

Taking into account a membership function of a linear type, trapezoidal fuzzy numbers together with triangular numbers are the most commonly used fuzzy numbers in numerical analysis. In this article, trapezoidal fuzzy numbers have been applied to find a numerical solution to the Boltzmann transport equations in the fuzzy form. 
A membership function of a trapezoidal fuzzy number $\widetilde{a}$ can be defined as follows (Giachetti and Young, 1997)

$$
\mu_{\tilde{a}}(x)= \begin{cases}\frac{1}{\sigma}\left(x-x_{0}+\sigma\right) & x_{0}-\sigma \leqslant x \leqslant x_{0} \\ 1 & x_{0} \leqslant x \leqslant y_{0} \\ \frac{1}{\beta}\left(x-y_{0}+\beta\right) & y_{0} \leqslant x \leqslant y_{0}+\beta \\ 0 & \text { otherwise }\end{cases}
$$

where $x_{0}$ and $y_{0}$ are two defuzzifiers - the left and the right one, respectively, $\sigma$ and $\beta(\sigma>0$, $\beta>0)$ are left and right fuzzinesses. A trapezoidal fuzzy number can be written as $\widetilde{a}=\left(x_{0}, y_{0}, \sigma, \beta\right)$ and illustrated as in Fig. 8 .

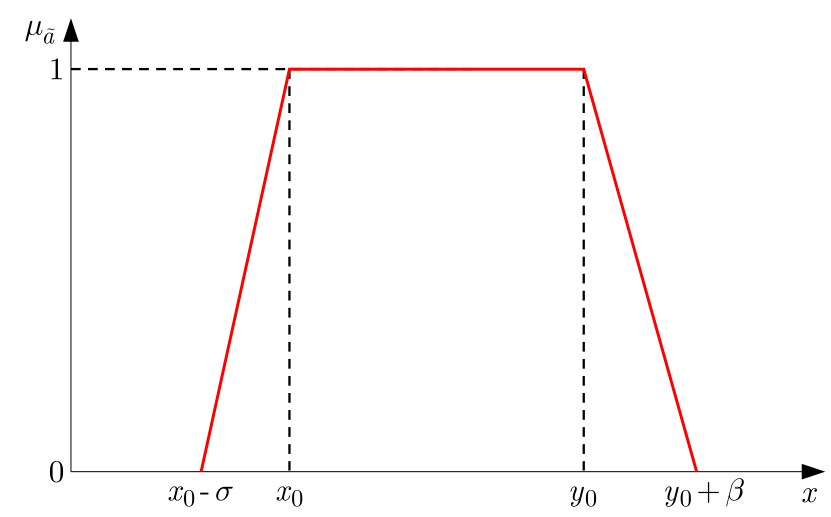

Fig. 8. Trapezoidal fuzzy number

Due to the fact that every $\alpha$-cut is a closed interval of real numbers, the mathematical operations can be defined using the rules of the directed interval arithmetic.

The $\alpha$-cut of a fuzzy number $\widetilde{a}$ can be written as (Guerra and Stefanini, 2005)

$$
\forall \alpha \in[0,1] \quad \tilde{a}_{\alpha}=\left[a_{\alpha}^{-}, a_{\alpha}^{+}\right]
$$

with the conditions

$$
\begin{array}{ll}
\text { 1. } & a^{-}: \quad \alpha \rightarrow a_{\alpha}^{-} \in \mathbb{R} \\
\text { 2. } & a^{+}: \quad \alpha \rightarrow a_{\alpha}^{+} \in \mathbb{R} \\
\text { 3. } & a_{\alpha}^{-} \leqslant a_{\alpha}^{+}
\end{array}
$$

where $a^{-}\left(a^{+}\right):[0,1] \rightarrow \mathbb{R}$ is a limited, monotonic, increasing (decreasing) function for every $\alpha \in[0,1]$.

Finally, every fuzzy number can be defined as a sum of all its own $\alpha$-cut

$$
\widetilde{a}=\sum_{\alpha \in[0,1]} \tilde{a}_{\alpha}
$$

while the $\alpha$-cut of a trapezoidal fuzzy number $\widetilde{a}=\left(x_{0}, y_{0}, \sigma, \beta\right)$ can be expressed as

$$
\forall \alpha \in[0,1] \quad \widetilde{a}_{\alpha}=\left[x_{0}-(1-\alpha) \sigma, y_{0}+(1-\alpha) \beta\right]
$$

For the $\alpha$-cut of two fuzzy numbers $\widetilde{a}$ and $\widetilde{b}$, the mathematical operations can be defined as in Table 3 (Piasecka-Belkhayat, 2011). 
Table 3. Mathematical operations

\begin{tabular}{|l|c|}
\hline Name of operation & Formula \\
\hline \hline addition & $(\widetilde{a}+\widetilde{b})_{\alpha}=\left[a_{\alpha}^{-}+b_{\alpha}^{-}, a_{\alpha}^{+}+b_{\alpha}^{+}\right]$ \\
\hline subtraction & $(\widetilde{a}-\widetilde{b})_{\alpha}=\left[a_{\alpha}^{-}-b_{\alpha}^{+}, a_{\alpha}^{+}-b_{\alpha}^{-}\right]$ \\
\hline $\begin{array}{l}\text { multiplication by } \\
\text { a scalar }(k \in \mathbb{R})\end{array}$ & $k \widetilde{a}_{\alpha}=\left[\min \left\{k a_{\alpha}^{-}, k a_{\alpha}^{+}\right\}, \max \left\{k a_{\alpha}^{-}, k a_{\alpha}^{+}\right\}\right]$ \\
\hline multiplication & $(\widetilde{a} \widetilde{b})_{\alpha}=\left[\min \left\{a_{\alpha}^{-} b_{\alpha}^{-}, a_{\alpha}^{-} b_{\alpha}^{+}, a_{\alpha}^{+} b_{\alpha}^{-}, a_{\alpha}^{+} b_{\alpha}^{+}\right\}, \max \left\{a_{\alpha}^{-} b_{\alpha}^{-}, a_{\alpha}^{-} b_{\alpha}^{+}, a_{\alpha}^{+} b_{\alpha}^{-}, a_{\alpha}^{+} b_{\alpha}^{+}\right\}\right]$ \\
\hline $\begin{array}{l}\text { inverse } \\
\left(0 \notin\left[a_{\alpha}^{-}, a_{\alpha}^{+}\right]\right)\end{array}$ & $\left(\frac{1}{\widetilde{a}}\right)_{\alpha}=\left[\frac{1}{a_{\alpha}^{+}}, \frac{1}{a_{\alpha}^{-}}\right]_{\alpha}$ \\
\hline $\begin{array}{l}\text { division } \\
\left(0 \notin\left[b_{\alpha}^{-}, b_{\alpha}^{+}\right]\right)\end{array}$ & $\left(\frac{\widetilde{a}}{\vec{b}}\right)_{\alpha}=\left[\min \left\{\frac{a_{\alpha}^{-}}{b_{\alpha}^{-}}, \frac{a_{\alpha}^{-}}{b_{\alpha}^{+}}, \frac{a_{\alpha}^{+}}{b_{\alpha}^{-}}, \frac{a_{\alpha}^{+}}{b_{\alpha}^{+}}\right\}, \max \left\{\frac{a_{\alpha}^{-}}{b_{\alpha}^{-}}, \frac{a_{\alpha}^{-}}{b_{\alpha}^{+}}, \frac{a_{\alpha}^{+}}{b_{\alpha}^{-}}, \frac{a_{\alpha}^{+}}{b_{\alpha}^{+}}\right\}\right]_{\alpha}$ \\
\hline
\end{tabular}

\section{Acknowledgments}

The studies have been conducted as a part of the research projects financed by the Silesian University of Technology, Faculty of Mechanical Engineering.

\section{References}

1. Bejan A., Kraus D., 2003, Heat Transfer Handbook, John Wiley \& Sons, New Jersey

2. Cattaneo C., 1958 A form of heat conduction equation which eliminates the paradox of instantaneous propagation, Comptes rendus de l'Académie des Sciences, 247, 431-433

3. Chen G., Borca-Tasciuc D., Yang R.G., 2004, Nanoscale heat transfer, [In:] Encyclopedia of Nanoscience and Nanotechnology, American Scientific Publishers, 7, 359-429

4. Chen J.K., Tzou D.Y., Beraun J.E., 2006, A semiclassical two-temperature model for ultrafast laser heating, International Journal of Heat and Mass Transfer, 49, 307-316

5. Dziatkiewicz J., Kuś W., Majchrzak E., Burczyński T., Turchan Ł., 2014, Bioinspired identification of parameters in microscale heat transfer, International Journal for Multiscale Computational Engineering, 12, 1, 79-89

6. Escobar R.A., Ghai S.S., Jhon M.S., Amon C.H., 2006, Multi-length and time scale thermal transport using the lattice Boltzmann method with application to electronics cooling, International Journal of Heat and Mass Transfer, 49, 97-107

7. Ghai S.S., Kim W.T., Escobar R.A., Amon C.H., Jhona M.S., 2005, A novel heat transfer model and its application to information storage systems, Journal Applied Physics, 97, https://doi.org/10.1063/1.1853896

8. Giachetti R.E., Young R.E., 1997, A parametric representation of fuzzy numbers and their arithmetic operators, Fuzzy Sets and Systems, 91, 185-202

9. Guerra M.L., Stefanini L., 2005, Approximate fuzzy arithmetic operations using monotonic interpolations, Fuzzy Sets and Systems, 150, 5-33

10. Hanss M., 2005, Applied Fuzzy Arithmetic, Springer-Verlag, Berlin-Heidelberg

11. Ho J.R., Kuo Ch.P., Jiaung W.S., 2003, Study of heat transfer in multilayered structure within the framework of dual-phase-lag heat conduction model using lattice Boltzmann method, International Journal of Heat and Mass Transfer, 46, 55-69 
12. Lee J.B., KAng K., LeE S.H., 2011, Comparison of theoretical models of electron-phonon coupling in thin gold films irradiated by femtosecond pulse lasers, Materials Transactions, 52, 3, $547-553$

13. Majchrzak E., Dziatkiewicz J., 2015, Analysis of ultashort laser pulse interactions with metal films using a two-temperature model, Journal of Applied Mathematics and Computational Mechanics, 14, 2, 31-39

14. Majchrzak E., KAŁużA G., 2015, Heat flux formulation for 1D dual-phase lag equation, Journal of Applied Mathematics and Computational Mechanics, 14, 1, 71-78

15. Majchrzak E., Mochnacki B., 2014, Sensitivity analysis of transient temperature field in microdomains with respect to the dual phase lag model parameters, International Journal for Multiscale Computational Engineering, 12, 1, 65-77

16. Majchrzak E., Mochnacki B., Greer A.L., Suchy J.S., 2009, Numerical modeling of short pulse laser interactions with multi-layered thin metal films, CMES: Computer Modeling in Engineering and Sciences, 41, 2, 131-146

17. McDonough J.M., Kunadian I., Kumar R.R., Yang T., 2006, An alternative discretization and solution procedure for the dual phase-lag equation, Journal of Computational Physics, 219, 163-171

18. Mochnacki B., Paruch M., 2013, Estimation of relaxation and thermalization times in microscale heat transfer model, Journal of Theoretical and Applied Mechanics, 51, 4, 837-845

19. Mochnacki B., PiaseckA-Belkhayat A., 2013, Numerical modeling of skin tissue heating using the interval finite difference method, MCB: Molecular and Cellular Biomechanics, 10, 3, 233-244

20. Отто K., Lewis A.D., Antonsson E., 1993, Approximation alpha-cuts with the vertex method, Fuzzy Sets and Systems, 55, 43-50

21. Piasecka-Belkhayat A., 2011, Interval boundary element method for imprecisely defined unsteady heat transfer problems, D.Sc. Dissertation, Gliwice

22. Piasecka-Belkhayat A., Korczak A., 2014a, Application of the interval lattice Boltzmann method for a numerical modeling of thin metal films irradiation by ultra short laser pulses, IAPGOŚ, 4, $85-88$

23. Piasecka-Belkhayat A., Korczak A., 2014b, Modelling of transient heat transport in one-dimensional crystalline solids using the interval lattice Boltzmann method, Recent Advances in Computational Mechanics, T. Łodygowski, J. Rakowski and P. Litewka (Edit.), Taylor \& Francis Group, A.A. Balkema Book, London, 363-368

24. Piasecka-Belkhayat A., Korczak A., 2016, Numerical modelling of the transient heat transport in a thin gold film using the fuzzy lattice Boltzmann method with $\alpha$-cuts, Journal of Applied Mathematics and Computational Mechanics, 15, 1, 123-135

25. Piasecka-Belkhayat A., Korczak A., 2017, Modeling of thermal processes proceeding in a 1D domain of crystalline solids using the lattice Boltzmann method with an interval source function, Journal of Theoretical and Applied Mechanics, 55, 1, 167-175

26. Tzou D.Y., 1997, Macro- to Microscale Heat Transfer: The Lagging Behavior, Taylor and Francis, Washington

27. Venkatakrishnan K., Tan B., Ngoi B.K.A., 2002, Femtosecond pulsed laser ablation of thin gold film, Optics and Laser Technology, 34, 199-202

28. Zhang Z.M., 2007, Nano/microscale Heat Transfer, McGraw-Hill, New York 